\title{
The case for sensorimotor coding in working memory
}

\author{
MARGARET WILSON \\ North Dakota State University, Fargo, North Dakota
}

\begin{abstract}
The highly influential Baddeley and Hitch model of working memory (Baddeley \& Hitch, 1974; see also Baddeley, 1986) posited analogical forms of representation that can be broadly characterized as sensorimotor, both for verbal and for visuospatial material. However, difficulties with the model of verbal working memory in particular have led investigators to develop alternative models that avoid appealing either to sensory coding or to motoric coding, or to both. This paper examines the evidence for sensorimotor coding in working memory, including evidence from neuropsychology and from sign language research, as well as from standard working memory paradigms, and concludes that only a sensorimotor model can accommodate the broad range of effects that characterize verbal working memory. In addition, several findings that have been considered to speak against sensorimotor involvement are reexamined and are argued to be in fact compatible with sensorimotor coding. These conclusions have broad implications, in that they support the emerging theoretical viewpoint of embodied cognition.
\end{abstract}

The past two decades have seen an explosion of interest in the topic of working memory. In the 1970s and 1980s, Baddeley and Hitch (1974; Baddeley, 1986) proposed that previous conceptions of short-term memory should be replaced by a multicomponent model of temporary information storage and manipulation, including a verbal component and a visuospatial component. Since then, the model-and in particular the "phonological loop" account of verbal rehearsal-has created a fertile domain of research, encompassing not only empirical confirmation and expansion of the model (see Baddeley \& Hitch, 1994, for review) but also applications to processes, such as language acquisition and reading (e.g., Ellis \& Sinclair, 1996; Gathercole \& Baddeley, 1993), and computational modeling of the proposed underlying mechanisms (e.g., Burgess \& Hitch, 1992, 1999; Henson, 1998; Page \& Norris, 1998).

(An additional recent trend has been the expansion of the concept of working memory to include the capacity for on-line processing [Carpenter, Miyake, \& Just, 1994] and semantic comprehension [Martin \& Lesch, 1996] and to explain the interactions between long-term knowledge and working memory capacity [Cantor \& Engle, 1993; Gathercole \& Martin, 1996; Nairne \& Kelley, 1999; Neely \& LeCompte, 1999; for general review, see Richardson et al., 1996]. For the purpose of this paper, which focuses on the storage and maintenance of information, the narrower usage of the term working memory will be retained.)

This work was partially supported by NSF Grant OSR 9452892. The author thanks Nelson Cowan, Karen Emmorey, Arthur Glenberg, and John Richardson for their valuable comments. Correspondence should be addressed to M. Wilson, Department of Psychology, North Dakota University, Fargo, ND 58105 (e-mail: margwils@badlands.nodak.edu).
However, in addition to the considerable empirical and theoretical successes created by the Baddeley model, a number of difficulties have emerged: In particular, several of the signature effects that underlie the original model have been found to behave in ways not originally predicted. Some researchers have sought to adapt the model to accommodate the new data, whereas others have pursued alternative approaches, and the past 5 years have seen a proliferation of competing models of working memory. Although the Baddeley model posits fairly peripheral forms of representation (visuospatial working memory preserves depictive properties of the stimulus, verbal working memory preserves phonological and articulatory properties), more recent models have shown a consistent trend away from sensorimotor representations. In this article, the viability of such a theoretical move is considered.

This question is of considerable theoretical importance because it bears on the concept of embodied cognition that is gaining currency within cognitive science (e.g., Clark, 1997; Glenberg, 1997; Johnson, 1987; Kutas \& Federmeier, 1998; Lakoff \& Johnson, 1999). The core insight of this viewpoint is that many of our "central cognitive" abilities may in fact be parasitic upon perceptual and motoric processes. One line of theorizing, also known as situated cognition, emphasizes the on-line use of overt sensorimotor activity to assist with cognitive tasks that interact with the world. Of equal interest, though, is off-line embodied cognition, in which sensorimotor processes are run covertly to assist with the representation and manipulation of information, in the temporary absence of task-relevant input or output. Such an arrangement would make sense, given our evolutionary heritage from creatures whose neural resources were devoted largely to perceptual and motor processes. Indeed, given that we have such resources, it would be odd if we did not exploit them 
Table 1

Presence of Effects in Verbal Working Memory Under Various Presentation Conditions

\begin{tabular}{ccccc}
\hline & \multicolumn{4}{c}{ Effect } \\
\cline { 2 - 4 } Presentation & Phonological & Irrelevant & Word & Articulatory \\
Conditions & Similarity & Speech & Length & Suppression \\
\hline
\end{tabular}

No Additional Manipulations

Auditory stimuli $\quad+$

Visual stimuli +

Articulatory Suppression

Auditory stimuli $\quad+$

Visual stimuli

$+$

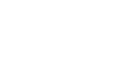

$+$

$+$

$+$

Irrelevant Speech

Auditory stimuli

Visual stimuli

+
$-?$

$\begin{array}{ll}+ & - \\ - & -\end{array}$

$-$

$\begin{array}{ll}-? & + \\ -? & +\end{array}$

whenever possible to assist and enhance off-line cognitive processing. The issue of sensorimotor involvement in working memory, then, goes beyond the question of how we perform immediate serial recall, to make contact with basic issues about the nature of our cognitive architecture.

\section{The Classic Data and the Rehearsal Loop Model}

Although visuospatial working memory has not yielded strong evidence regarding its internal structure, beyond its analogical nature, verbal working memory from the beginning showed effects and interactions that demanded explanation. The pattern of data that led to the concept of a phonologically based rehearsal loop in working memory has been frequently reviewed (e.g., Baddeley, 1990; Gupta \& MacWhinney, 1995; Neath, Surprenant, \& LeCompte, 1998; Wilson \& Emmorey, 1997a) and is presented here only in brief. That data pattern involves four effects on immediate serial recall: the phonological similarity effect (poor recall of similar-sounding items); the word length effect (poor recall of long items); the articulatory suppression effect (poor recall when there is competing activity of the articulators); and the irrelevant speech effect (poor recall when there is competing auditory input). The immediate suggestion these data seem to make is that verbal materials are coded in working memory in something like their "surface form"-either what they sound like or how we would pronounce them.

In addition to these effects that suggest coding in a speech-like form, interactions among the effects suggest a dissociation between two components within verbal working memory (see Table 1 for a summary of interactions). Articulatory suppression eliminates the phonological similarity effect, but only when the target items are presented in an indirect, nonphonological form (print or nameable pictures). When items are presented in phonological form (auditory speech), the phonological similarity effect occurs even under articulatory suppression. This suggests that materials that arrive already in phonological form will automatically result in phonological confu-

sions; however, translating materials into the appropriate phonological code requires some form of mental articulation. When this articulation is blocked, the phonological code is never achieved, and so phonological confusions will not take place. In addition, articulatory suppression eliminates the word length effect regardless of how materials are presented. This suggests that, unlike the similarity effect, the length effect is a direct consequence of some articulatory process: When articulation is blocked, the length effect cannot occur.

(It is worth noting that neither articulatory suppression nor irrelevant speech reduces performance to zero. This requires explanation-particularly since, as described above, articulatory suppression is capable of completely eliminating other signature effects. An immediately obvious interpretation is that other resources are available for maintaining information. A few candidates are visual representation of the physical form of the word, visual representation of the meaning of the word [particularly for concrete words], and abstract semantic representation. None of these appears to be a particularly robust strategy for maintaining multiple items in order, which accounts for the ubiquitous use of phonological representation in these tasks.)

Thus, these data have been taken as indicating a twopart system, consisting of a buffer that stores information in phonologicalform and an articulatory rehearsal process that can be used to load or refresh the buffer (Baddeley, 1986). As Table 1 shows, the effects associated with the buffer - the phonological similarity and irrelevant speech effects - are disruptable with visual presentation but not with auditory presentation, whereas the effects associated with articulation-the word length and articulatory suppression effects-stand or fall regardless of modality (for further evidence of two distinct components, see Longoni, Richardson, \& Aiello, 1993, and Schweickert, Guentert, $\&$ Hersberger, 1990). This two-part system has been called the articulatoryloop, or, more recently, the phonological loop.

Although this basic model does not involve strong commitments on the exact nature of these two components, it is worth noting their immediate resemblance to speech perception and speech production, respectively. Of interest here is a general class of explanation that appeals to some form of quasi-sensory coding, on the one hand, and quasi-motoric coding, on the other hand. Either or both of these may be fairly peripheral, representing more or less faithfully the specifics of sensory input and motor output; or they may be more abstract, corresponding to relatively late perceptual processing and relatively early response planning. Nevertheless, such representations would still retain the characteristic of being modality specific (i.e., tied to a particular sensory or response modality and retaining some of the analogical representation features of that modality). Such an account will be referred to here as a sensorimotor account, on the understanding that ex- 
actly how peripheral the representations are remains an open question.

\section{The Move Away From \\ Sensorimotor Involvement}

As further research on visuospatial working memory has continued (see Logie, 1995, for review; see also recent brain imaging studies, e.g., Awh et al., 1999), it remains widely accepted that this component is an analogical, quasi-sensory (and perhaps also quasi-motoric) form of representation. This is due in part to the convergence of this literature with the literature on visual and spatial imagery, which in the past three decades has produced overwhelming evidence for the analogical nature of imagery (reviews of which can be found in most undergraduate cognition textbooks). In contrast, the phonological loop model has attracted controversy on this point.

In recent years, a number of difficulties with the concept of the phonological loop have arisen, which have spurred the development of alternative models. To begin with, certain specific claims associated with the Baddeley model have not been supported. For example, the suggestion that a main function of the phonological loop is to aid speech processing has met with disconfirming evidence, at least for sentences of ordinary length and complexity (Howard \& Butterworth, 1989; Martin, 1995; Martin \& Romani, 1994; Martin, Shelton, \& Yaffee, 1994). (A more recent suggestion, that one function of the loop is to aid the learning of new vocabulary, has been more successful; for review, see Baddeley, Gathercole, \& Papagno, 1998.) A second suggestion, that the phonological loop is limited strictly to phonological materials, has also been called into question. It appears instead that nonphonologicalmaterials can produce the "irrelevant speech effect" (or "irrelevant sound effect," as the authors more accurately call it [e.g., Jones \& Macken, 1993; Jones, Macken, \& Murray, 1993], although the effect may be smaller for nonspeech than for speech [LeCompte, Neely, \& Wilson, 1997]). Furthermore, there is evidence suggesting that nonphonological auditory materials can be rehearsed using the same system as that used for rehearsing speech (Keller, Cowan, \& Saults, 1995; Pechmann \& Mohr, 1992).

These findings can be accommodated with fairly minor revisions. More serious are findings that appear to undermine the core assumptions of the model. One major difficulty is raised by the irrelevant speech effect. It turns out that listening to irrelevant speech fails to disrupt the similarity effect, at least with auditory list presentation (Boyle \& Coltheart, 1996; Surprenant, Neath, $\&$ LeCompte, 1999). This appears to contradict a fundamental assumption of the model-namely, that the locus of both these effects (irrelevant speech and phonological similarity) is one and the same, the storage buffer. In contrast, there is evidence that irrelevant speech may disrupt the word length effect (Neath et al., 1998; but see Tremblay, Macken, \& Jones, 1999). Again, this is surprising, since the locus word length effect is allegedly not the buffer, but the articulatory rehearsal process.
A second major difficulty is raised by the word length effect, which various lines of evidence suggest may not be due to articulatory processes. For example, congenital anarthrics (people unable to speak for neurological reasons; e.g., cerebral palsy), who have never spoken intelligibly, nevertheless show a word length effect (Bishop \& Robson, 1989). The word length effect has also been challenged on the grounds that it may be an effect of phonological complexity rather than articulation time (Caplan, Rochon, \& Waters, 1992).

In the face of these growing challenges, investigators have proposed a number of alternative models. Notably, in response to the data from anarthric subjects reported by Bishop and Robson (1989), several authors have proposed that motoric or articulatory processes may not, in fact, be a key component in verbal working memory. Gathercole and Martin (1996), for example, propose that the representations involved in rehearsal are representations of vocal gestures that are intended for speech perception, not speech production. These representations are considered to be long-term memory representations, and working memory is considered to consist of their temporary activation. Other recent proposals have instead suggested that item representations in verbal working memory are not be tied to any sensory modality (e.g., Jones, Beaman, \& Macken, 1996).

However, there is little agreement in the field about the details of a model that would successfully replace the traditional account. In general, models that omit reference to sensory coding and/or articulatory processes must find a way to account for the divergent patterns of data observed for visual stimuli (print) versus auditory stimuli (speech). These differences include not only those described above (see Table 1) but also the modality effect (a large recency effect for speech but not print) and the suffix effect (disruption of the recency effect by an irrelevant final stimulus, for speech but not print). If these differences are not to be explained in terms of an articulatory process required for recoding print, then some alternative explanation must be offered. Likewise, if there is no modalityspecific sensory coding, this would presumably leave print and speech on equal footing, and, again, the observed differences must be explained.

One approach to solving this question is taken by the temporal distinctiveness theory, according to which retrieval from working memory consists of temporally defined search without involvement of articulatory rehearsal (Glenberg \& Swanson, 1986; LeCompte, 1996; Marks \& Crowder, 1997). Errors in retrieval from working memory are thought to result from interference between items that fall within the same temporal search set. In order to account for differences between print and speech stimuli, it is proposed that auditory items are coded with greater temporal precision than visual items.

A different approach is taken by the feature model (Nairne, 1990; Neath, 2000), in which errors are caused by overwriting of features. Items thus interfere with one another within working (or primary) memory, and recall 
consists of an attempt to match these corrupted traces to items within a particular search set in long-term (or secondary) memory. The features that are subject to overwriting in this fashion can be either modality specific or modality independent. Auditory stimuli are claimed to possess features of both types, whereas visual stimuli are claimed to possess few modality-specific features. (It is worth noting that although this model differs in important respects from a sensorimotor model-most notably, by excluding articulation-it carves up the territory in a similar way. Modality-specific features, according to the feature model, come from sensory input and are primarily associated with the auditory modality. Modalityindependentfeatures, on the other hand, are those features that are internally generated, resemble "inner speech," are disrupted by articulatory suppression, and can be had from print presentation.)

Yet another approach is the object-oriented episodic record model (Jones et al., 1996; Jones \& Tremblay, 2000). In contrast to the two theories described above, which incorporate modality-specific input representations, this model proposes that items are held in working memory in an abstract, modality-neutral form. This form of coding is specialized for the retention of serial order, and incoming materials such as irrelevant speech, which have "changing state" properties, are specifically disruptive of memory for serial order. Although both auditory and visual stimuli can be recoded into this amodal form, auditory stimuli are thought to have a privileged ability to disrupt the serial order information of previously encoded materials, as shown by the irrelevant speech (or irrelevant sound) effect.

In short, there is little agreement in the field on how best to model verbal working memory. Nevertheless, the appeal of these various accounts is clear. They offer ways to circumvent the troubles of the phonological loop, particularly the unpredicted behavior of the length effect and the irrelevant speech effect. In general, they do so by omitting sensory coding, motor coding, or both. However, I will argue that data from neuropsychology, from the study of sign language, and from the further study of the classic effects in working memory make it increasingly difficult to form a coherent theoretical account that does not involve both sensory and motor coding.

\section{Is the Length Effect Due to Articulation Time?}

One of the most important bases of the argument for articulatory rehearsal has been the word length effect. However, a number of investigators have questioned whether the length effect necessitates an articulatory account. For example, it has been shown that a length effect can result from the delay caused by long items during spoken output (e.g., Avons, Wright, \& Pammer, 1994; Cowan et al., 1992). This might suggest that the length effect is actually an artifact of the reporting process, rather than a consequence of how materials are maintained in memory. However, this cannot fully account for the length effect, since the effect occurs with other forms of report (e.g., a probe paradigm [Avons et al., 1994] or a written report using abbreviations for long items [Baddeley, Lewis, \& Vallar, 1984]).

Alternatively, it has been suggested that the effect may be due simply to the temporal length of the trace created by the stimulus. Brown and Hulme (1995) report a computer simulation that produces a temporal length effect purely on the basis of trace decay. However, this result depends on the temporal duration of the stimulus as presented and thus cannot explain the length effect for printed words presented at a fixed rate.

Alternatively, it has been suggested that the length effect may be due to phonological structure (e.g., differences in the number of syllables or phonemes), rather than constraints of articulation itself. If so, then the "length" effect would appear to be due to fairly abstract phonemic representations and possibly not to a time-based articulatory process at all. Baddeley, Thomson, and Buchanan (1975) found a word length effect for long versus short words that were matched for number of syllables and phonemes and differed only in the articulation length of the phonemes themselves, whereas Caplan et al. (1992) and Service (1998) failed to replicate this result. Caplan et al. argue that although rehearsal does "require some form of phonological output planning," this output planning does not fully specify articulatory gestures. Instead, they conceive of this output planning as activating "underspecified lexical phonological representations." Their data clearly open the door, though, to a stronger interpretation in which output planning plays no role at all. Baddeley and Andrade (1994) and Caplan and Waters (1994) offer further debate on whether a length effect occurs for phoneme-controlled stimuli. It is difficult, though, to adjudicate between the conflicting findings, given the small differences in temporal duration that remain when syllables and phonemes are controlled.

Evidence that may bear on this issue comes from a length effect observed in working memory for sign language, which growing evidence indicates is governed by the same principles as working memory for spoken language (see below). Because the sublexical structure of signs has many properties similar to the phonology of spoken words, the term phonology has been broadened to apply to signed languages as well as spoken languages (e.g., Corina \& Sandler, 1993; Coulter, 1993). Thus, signs can be analyzed in terms of their sublexical phonological structure. However, because of the large-scale motor movements in sign language, it is easier to detect a length effect independent of number of phonemes or syllables.

Using a probe paradigm, Wilson and Emmorey (1998b) report a length effect for signs from American Sign Language in deaf native signers, which cannot be explained in terms of number of sublexical units within the signs. 
The short signs used by Wilson and Emmorey actually contained, on average, more linearly sequenced phonological segments per sign than the long signs (segment counts were made using Sandler's, 1989, model). This is because short signs in American Sign Language frequently use a double-tap movement. Signs can also be analyzed as monosyllabic or bisyllabic (e.g., Brentari, 1990), but the lists of short signs and long signs used by Wilson and Emmorey were matched for number of monosyllabic and bisyllabic signs. Finally, if considered purely in terms of movement trajectories, independent of any linguistic analysis, the short signs on average contained more distinct trajectories than the long signs, again because of repeated tapping and brushing movements in the short signs. Instead, the long signs were long in the temporal sense simply because they covered greater distances. Because of the large-scale motor movements involved, this difference in temporal length is large enough to unambiguously create a length effect. Thus, unless one argues that working memory for sign language is governed by a different set of principles than working memory for spoken language (and, at the moment, there is little reason to suspect this), the data from sign language suggest that the length effect is indeed an effect of articulation time.

Further evidence comes from a length effect for spoken language when articulation rate is controlled by explicit instructions. Cowan and colleagues (Cowan, Nugent, Elliott, \& Geer, 2000; Cowan, Wood, Nugent, \& Treisman, 1997) asked subjects to mentally rehearse items either fast or slow. This artificially induced articulation rate produced a standard length effect, with "slow" items showing worse performance than "fast" items.

Furthermore, the very idea that number of phonemes could account for the length effect has been called into question. Brown and Hulme (1995) argue that when a subject tries to reconstruct a partially decayed or corrupted item, items with many phonemes will actually fare better than items with few. A single phoneme (or even several phonemes) missing from the word hippopotamus, for example, will be less problematic than a single phoneme missing from the word cat. In the classic word length effect, then, polysyllabic words presumably produce worse performance because their pronunciation time is considerably longer than that for monosyllabic words, and this temporal length effect overrides the benefit of more numerous phonemes. (Tentative empirical support has been found for this point by Cowan et al., 1997. They found that when articulation rate is controlled by explicit instructions, as described above, bisyllabic words result in better performance than monosyllabic words, the reverse of the usual pattern. However, this finding was in an experiment where subjects were required to perform backward report. Cowan et al., 2000, failed to replicate this finding with forward report.)

However, the word length effect is not the only source of evidence for the role of articulation. Further support comes from a variety of experimental paradigms that examine the limits imposed by the physical demands of articulation.

\section{Constraints Imposed by \\ Articulatory Limitations}

One source of evidence that memory span is limited by how quickly material can be articulated comes from cross-linguistic data. Span for digits, for example, has consistently been found to be greater in languages whose digits are short and can be pronounced quickly (Ellis \& Hennelly, 1980; Stigler, Lee, \& Stevenson, 1986). This cross-linguistic difference has even been found within individuals. In a study of Welsh-English bilinguals, memory span was found to be longer for English than for Welsh within the same subject sample (Ellis \& Hennelly, 1980). Differences in articulation rate can also account for the fairly extreme difference in span between spoken and signed languages. Typical linguistic memory span for deaf signers is around four items, in contrast to approximately seven items for speakers. However, this difference in span appears to reflect differences in articulation time. Although the two modes of language are equally fast sentence by sentence (due to the efficiency of spatialized grammar in sign language), individual signs take approximately twice as long to articulate as individual spoken words (Klima \& Bellugi, 1979). And when span is divided by articulation rate (see below), approximately equal time limits are found for signed and spoken language (Marschark, 1996).

Further evidence comes from the correlation between articulation rate and span across individuals. In a typical experiment, the subject is asked to articulate single words as quickly as possible, to obtain a measure of that individual's maximum articulation rate. This measure is then compared with the subject's verbal memory span. Even when age is controlled or partialed out, significant correlations between articulation rate and span are found (see Gathercole \& Baddeley, 1993, for review). (The relation does not hold for very young children [Cowan et al., 1994; Gathercole, Adams, \& Hitch, 1994], but this is consistent with other evidence that very young children do not always spontaneously engage in articulatory rehearsal.) Furthermore, there appears to be a roughly constant time limit on memory rehearsal, with some variation across individuals, estimated at just less than $2 \mathrm{sec}$ by dividing span (number of items) by articulation rate (items per second) (see Schweickert \& Boruff, 1986, for review).

One concern is that the correlation might be due to some more general factor, such as individual differences in cognitive processing speed. However, Cowan et al. (1998) found that there are two distinct processing speeds that appear to contribute to working memory performancearticulation rate, and rate of retrieval from working memory-which are both related to memory span but are independent of one another. This suggests that the correlation between articulation rate and span represents a fairly specific contribution of articulatory processes, rather than general cognitive capabilities.

A second concern is that the word length and phonological similarity effects have been observed with visual presentation rates too fast for overt articulation (Coltheart 
\& Langdon, 1998; LeCompte, Speed, \& Marks, 2000). This would seem to suggest that articulation rate cannot be responsible for the length effect or for the recoding of print into phonology. However, although rehearsal speed tracks overt articulation speed, this does not necessitate that the two processes operate at the same speed. A kind of "dry-run" covert execution of motor programs could easily take less time than overt execution and yet still be proportional to the time required for overt execution. This account of the data is lent plausibility by the fact that, even with rapid presentation, both effects are eliminated by articulatory suppression.

In addition, we should note that not every item in a rapid stream need be successfully recoded in order to produce recoding-based effects such as the word length effect. For example, in a rapid stream of eight words, a subject might have time to "pluck out" four items and recode them using articulation. But if phonologically longer words are used as stimuli (holding constant the presentation time of each word), the subject may be able to recode only two or three items. Thus, a word length effect can emerge, based on articulatory recoding, despite the fact that it is not possible for the subject to articulate all of such a rapid list.

A final source of evidence comes from children who consistently misarticulate particular phonemes, such as substituting /w/ for / r /. In a study by Locke and Kutz (1975), such children had no difficulty correctly hearing and understanding words that use these phonemes. But when asked to perform a short-term memory task, these children committed / w/-/r/ substitutions (e.g., wing for ring). Recall consisted of pointing to pictures, so the effect was not due to misproduction of the target word at time of report. Instead, it appears that the subjects' atypical articulatory programs affected the identity of items held in working memory. This finding provides compelling support for the role of articulatory processes in rehearsal.

\section{Is Articulatory Suppression}

\section{Just a Form of Irrelevant Speech?}

In addition to the word length effect, any theory that does not invoke articulatory processes must explain the effect of articulatory suppression. One appealing possibility is to explain suppression in terms similar to irrelevant speech. This can take several forms:

1. One approach is to explain both effects in terms of auditory input (cf. Gupta \& MacWhinney, 1995). The speech sounds created by the subject's articulation of an irrelevant word reenter the system through the subject's own ears, creating an irrelevant speech effect.

2. A second approach is to posit that articulatory activity, regardless of whether it produces external sound, can internally generate a quasi-perceptual representation (cf. J. D. Smith, Reisberg, \& Wilson, 1992). This representation then functions equivalently to irrelevant speech and accounts for the disruptive effects of articulatory suppression. (A more lenient version, under which articulatory suppression can indeed generate covert "irrelevant speech" but also has direct effects on an articulatory rehearsal process, will be considered later.)

3. A third approach holds that both irrelevant speech and articulatory suppression give rise to abstract, modalityneutral phonological representations that are neither perceptual nor productive in nature and that interfere with modality-neutral representations of the to-be-remembered material (cf. Jones et al., 1996).

However, a number of considerations argue against this general approach to articulatory suppression. First, silent articulation reduces performance (Gupta \& MacWhinney, 1995; Macken \& Jones, 1995). (The effect is not as large as for vocalized articulation, but there are reasons to believe that this is because activity of the vocal cords is one of the relevant aspects of articulatory rehearsal; J. D. Smith et al., 1992, p. 106.) This finding eliminates the first possibility listed above, that the effect of articulatory suppression is due entirely to the external auditory stimulation it creates. In addition, even non speech mouth movements, such as chewing or clamping one's jaws and lips shut, can produce the articulatory suppression effect (see J. D. Smith, Wilson, \& Reisberg, 1995, for review), which is difficult to reconcile with the second and third possibilities. Presumably, these mouth movements do not correspond to any representations that could mimic irrelevant speech or irrelevant sound.

An additional consideration is that, on any of the accounts listed above, the effects of irrelevant speech should be virtually identical to those of articulatory suppression (with the possible exception that articulatory suppression may be more distracting, because it requires some degree of control by the subject; cf. Neath et al., 1998). However, we know that the two manipulations do not produce identical effects. For example, as Baddeley (2000) points out, a single repeated item can be used to create successful articulatory suppression but does not create an irrelevant speech effect.

A further difficulty comes from the way the two effects interact with each other. If they have their effects for the same reasons, then we would expect their effects either to generally be additive or perhaps to generally be subadditive if there is an easily reached upper limit on the amount of disruption that can be created in this manner. What we would not expect is for the two effects to interact in some circumstances but not in others. Yet this is what in fact occurs. The effect of irrelevant speech is eliminated by articulatory suppression with print presentation of the to-be-remembered materials (Salamé \& Baddeley, 1982), but not with auditory presentation (Gupta \& MacWhinney, 1995; Hanley \& Broadbent, 1987). In other words, the behavior of the irrelevant speech effect is the same as that of the phonological similarity effect (see Table 1). This finding is difficult to explain if articulatory suppression and irrelevant speech are functionally equivalent, but it is easy to explain if the one disrupts an articulatory process that feeds a phonological store and the other directly disrupts the store. When materials are presented as print and articulation is blocked, the materi- 
als never arrive in the store, and, hence, irrelevant speech should not matter.

Nevertheless, recent findings do appear to suggest a striking equivalence between articulatory suppression and irrelevant speech. It has been reported that irrelevant speech disrupts the phonological similarity effect for visual lists but not auditory lists, just as articulatory suppression does (Surprenant et al., 1999; but see Boyle \& Coltheart, 1996; see also criticisms by Baddeley, 2000), and, furthermore, that irrelevant speech does disrupt the word length effect, just as articulatory suppression does (Neath et al., 1998; but see Tremblay et al., 1999). These results, if they hold up, contradict what has been taken as a core prediction of the sensorimotor account: The similarity effect is identified with the perceptual store and should therefore be disrupted by perceptual competition but not articulatory competition; the word length effect, in contrast, is identified with the articulatory process and should be disrupted by articulatory competition but not perceptual competition.

However, this line of reasoning is predicated on the assumption that task-irrelevant representations, whether perceptual or articulatory, do not spread from component to component within the phonological loop. According to this assumption, irrelevant material that enters directly into the buffer (irrelevant speech) remains solely in the buffer and has no impact on articulatory rehearsal, whereas irrelevant material that enters the system via articulation (articulatory suppression) remains solely in the articulatory rehearsal process and has no impact on the buffer. This assumption has gone virtually unchallenged in the working memory literature; yet, once brought to the fore, the assumption appears not only unmotivated but also highly improbable. Evidence suggests that maintenance rehearsal (at least for a subspan load) is relatively automatic, involving little in the way of executive control, beyond simple initiation of the process (e.g., Hitch \& Baddeley, 1976). If this is correct, there will be little opportunity to be selective about what items in the buffer will be rehearsed or what items being rehearsed will arrive in the buffer. Once the use of the loop has been set underway, it will proceed in an automatic fashion and will not be able to selectively exclude unwanted materials. In short, articulatory suppression may produce many effects similar to those of irrelevant speech, because both involve the generation of task-irrelevant representations that are then free to propogate throughout the verbal working memory system.

One testable implication is that articulatory suppression and irrelevant speech will diverge when the material is not phonological. For example, irrelevant speech may eliminate the word length effect, but other irrelevant sounds should not. These sounds can enter into the buffer, but, since they are not human vocal sounds, they will not be rehearsed. This prediction is borne out by the findings of Neath et al. (1998): Irrelevant tones reduce overall performance but spare the word length effect.

Finally, it is worth noting the odd theoretical tension in an account that denies articulatory involvement in work- ing memory yet holds that articulation is equivalent to irrelevant speech. Such a theory is committed to the claim that articulatory activity generates an internal representation in the same format as to-be-remembered materials, that this generation process is automatic and obligatory whenever articulation occurs, but that this process is not employed as a memory device.

\section{Could Articulatory Mechanisms Be Innate?}

Despite the considerable evidence in favor of articulatory involvement, though, there is still one major piece of evidence that speaks against such an account. This is the finding that congenital anarthrics show a word length effect (Bishop \& Robson, 1989), a finding that has caused numerous investigators to conclude that the word length effect is not caused by articulation. We should note, though, that the data do not force this conclusion, provided one accepts the admittedly counterintuitive hypothesis that articulatory programs are innately specified (or can be acquired on the basis of perception alone, which implies innate connections between perception and production). Before discarding the assumption of articulatory involvement, then, we should consider just how implausible such an innateness hypothesis really is.

In fact, there is growing evidence that something like a "body schema," with connections to both perception and production, is innately specified. Scatena (1990) reports that certain individuals with congenitally absent limbs experience vivid and well-formed phantom limbs. In addition, Meltzoff and Moore (1995) report that babies less than $1 \mathrm{~h}$ old will imitate the facial gestures of adults. This implies that the neonate possesses implicit knowledge of the connection between the perceived gesture, on the one hand, and the muscle movements necessary to produce a parallel gesture, on the other. Furthermore, Meltzoff and Moore report that infants with a condition that hinders tongue protrusion show signs of effort and frustration when viewing an adult face performing tongue protrusion. That is, the infant appears to be aware of the difference between the gesture he/she is attempting to imitate and the gesture that he/she actually produces.

It is not out of the realm of possibility, then, that congenital anarthrics possess rudimentary articulatory programs that could be recruited for rehearsal. This proposal becomes still more plausible when we consider that such articulatory programs need not contain much fine-grained detail in order to produce a length effect. For the oneversus three-syllable words used by Bishop and Robson (1989), even a sequence of one versus three undifferentiated syllables, or "beats," would be sufficient to produce a length effect (although some degree of identifying detail would probably be needed to make rehearsal a preferred strategy in the first place).

Since the innateness account is not out of the realm of possibility, its plausibility must be weighed against the body of evidence for articulatory involvement in deciding whether the data from anarthria call for a move away from articulation. 


\section{Modality Specificity: \\ Evidence From Sign Language}

Models of working memory are further constrained by evidence from sign language, which provides a unique opportunity to investigate the issue of modality-specific coding. A recent body of evidence indicates that the overall pattern of data for sign language is highly similar to that for speech. There is, for example, a handshape similarity effect, a sign length effect, a manual articulatory suppression effect, and an irrelevant sign effect, all of which seem to provide fairly precise parallels to the effects found for speech (Krakow \& Hanson, 1985; Poizner, Bellugi, \& Tweney, 1981; Wilson \& Emmorey, 1997a, 1997b, 1998a, 1998b). Furthermore, the pattern of interactions among these effects that are found for speech hold for sign language as well. When stimuli are presented in phonological form (videotaped signs), deaf signers show a similarity effect even under articulatory suppression. But when presentation is nonphonological (nameable pictures), deaf signers show the similarity effect only when there is no suppression-under suppression, the similarity effect vanishes (Wilson \& Emmorey, 1997a). (The presence of the similarity effect when there is no suppression indicates that the pictures are in fact being recoded into sign-based form.) In contrast, the length effect is abolished by suppression even when presentation is phonological (Wilson \& Emmorey, 1998b). This suggests that, whatever the structure of "verbal" working memory is, it is the same across languages based in different sensory modalities. In this respect, then, the data could be seen as supporting an abstract, amodal form of coding. But, given this overall pattern of parallel structure, it is critical to note that working memory structures for the two language modalities differ in certain respects that are linked to precisely the ways in which vision and audition differ.

In particular, it appears that speech-based memory encodes serial order in terms of time, whereas sign-based memory may be able to encode serial order in terms of space. For example, deaf individuals who are native signers of American Sign Language perform equally well on backward report as on forward report (Mayberry \& Eichen, 1991; Wilson, Bettger, Niculae, \& Klima, 1997). This is in striking contrast to the standard finding for hearing subjects, for whom backward report is a considerably more difficult task than forward report. Indeed, the deaf subjects in the Wilson et al. study outperformed hearing subjects on backward report, despite greater forward span in the hearing subjects. This indicates that the equal performance in the two conditions by the deaf subjects is not a floor effect, nor the result of a failure to retain serial order information. To be able to perform backward report at levels above that of hearing subjects, these deaf subjects must be retaining serial order information and retaining it in some form that is amenable to the task of reversing the order. In fact, spatial ordering, which is not only physically possible in signed languages but is actually incorporated into the grammar, could provide exactly such a form of reversible serial ordering. Items that are arrayed across space, unlike items arrayed across time, do not have a necessary directionality.

This explanation is lent plausibility by observations from several of the experiments cited previously, in which some deaf signers spontaneously reported the tobe-remembered items in a sequence of spatial locations, usually arrayed left to right. Furthermore, this spatial ordering appeared to be playing a functional role in memory. Some subjects used the spatial ordering as a mechanism for indexing the serial position of specific items-for example, by returning to a location to make a correction. More recently, we have found that memory span is lower for "fixed location" signs, which are anchored to a particular part of the body and cannot be rehearsed spatially, than for "neutral space" signs, which are formed in front of the torso and allow spatial rehearsal (Wilson \& Emmorey, 1998a).

We must ask, of course, whether this spatial ordering actually forms an integral part of sign-based rehearsal or whether it is a supplemental strategy that would be available to anyone regardless of the modality of their language - a kind of short-term memory analog to the method of loci. In fact, this appears not to be the case. Hearing subjects performing immediate serial recall do not benefit from words being associated with locations in space and may even be disrupted by it (Li \& Lewandowski, 1993, 1995; Serra \& Jonas, 1996). Instead, the evidence reviewed above seems to indicate that working memory for sign language and working memory for speech are each shaped and constrained by the specific capabilities of the sensory and/or motor modalities in which they are grounded.

To emphasize the relevance of these findings for models of verbal working memory, it is worth reiterating the high degree of parallel structure in every other respectthe effects of similarity, length, and suppression, and the ways in which their interactions depend upon mode of presentation-which suggests that we are observing a single set of organizational principles expressed in two modalities. As I have argued elsewhere (Wilson et al., 1997; Wilson \& Emmorey, 1997a, 1997b, 1998b), the phonological loop is not an innately specified mechanism, hard-wired for spoken language. Instead, this kind of rehearsal loop structure develops within a particular sensory and motor modality in response to suitably structured input. Linguistic structure, whether spoken or signed, appears to be sufficient to cause this kind of structure to emerge within working memory, whether in the auditory or the visual modality. We can thus consider speech-based working memory to be a specialization of more general auditory working memory and consider sign-based working memory to be a specialization of visuospatial working memory. (This proposal is similar in spirit to Glenberg's, 1997, argument that working memory should be conceptualized not as a set of preexisting modules but as a set of skills.)

Further evidence on modality specificity comes from a finding of an "irrelevant sign effect" (Wilson, Iverson, 
\& Emmorey, 2001). Deaf subjects who are asked to perform serial recall of signs are disrupted by the presence of meaningless but phonologically legal "pseudosigns" during a retention interval. In addition, just as in the case of speech memory, disruption also occurs when the irrelevant material is nonlinguistic-in this case, moving shapes. Hearing subjects, in contrast, are not disrupted by either form of irrelevant material while performing serial recall of printed words. On the sensorimotor account, this contrast occurs because signs are maintained in a sign-based code, whereas printed words are converted to a speech-based code.

This finding has important implications for theoretical accounts of the irrelevant speech effect. Any theory that posits amodal representation must explain why verbal working memory is not generally disrupted by irrelevant visual input. (Published studies on irrelevant visual input in verbal working memory are sparse. Indeed, the point is largely carried by this absence: There is a huge literature on the irrelevant sound effect but none on any "irrelevant visual stimulus effect" for verbal working memory. Two papers demonstrating the absence of an effect are Wilson et al., 2001, and Quinn \& McConnell, 1996.) How can one explain this absence in an amodal account of verbal working memory? One plausible move is to appeal to the many inherent differences between audition and vision. Relevant differences might include differing attentional filtering capabilities or differing robustness and duration of precategorical sensory storage. Indeed, as we have seen, several authors have suggested that visual stimuli result in representations in working memory that are less robust, are less temporally distinctive, or contain less modalityspecific information than auditory stimuli. Irrelevant visual stimuli, on this type of account, are simply not capable of disrupting working memory in the same way that auditory stimuli are. However, such an account is difficult to sustain in light of the sign language data. Visual stimuli clearly are capable of producing disruption within linguistic working memory, but this disruption occurs only for a language that is based in the visual modality.

What, then, are we to make of the contrasting results found for print stimuli? Why do print stimuli, which are visual, not behave like sign language stimuli-for example, by showing disruption from visual input? As suggested above, considerable evidence shows that print stimuli are not retained in working memory in visual form but are instead recoded into phonological form for maintenance (see Baddeley, 1986, for review). This overwhelming bias against maintaining print stimuli in visual form, in sharp contrast to the case of sign language, may be due to print's status as a derived code for language. Printed language is not a phenomenon that occurs on its own but instead is always secondary to, and built on, an expressive form of language such as speech or signing, which is the primary and native language of some human linguistic community. (For further discussion of print as a derived code, see Liberman, 1996, Shand \& Klima, 1981, and Wilson \& Emmorey, 1997b.) Thus, comparisons be- tween speech stimuli and print stimuli cannot be used to draw general conclusions about differences between the auditory and visual modalities. When instead we equate the two modalities by testing them both using stimuli that are primary, expressive forms of language, we find that auditory input and visual input do behave equivalently but that the effects of each are restricted to their own modality.

\section{Evidence From Neuropsychology}

Finally, the case for sensorimotor coding is supported by a growing body of data from neuropsychology (for reviews, see Baddeley, 1995; Howard \& Franklin, 1993; Jonides \& E. E. Smith, 1997; Paulesu, Bottini, \& Frackowiak, 1997; E. E. Smith, 2000; E. E. Smith \& Jonides, 1997; Vallar \& Papagno, 1995; and Vallar \& Shallice, 1990). In the domain of visuospatial working memory, brain imaging studies with humans strongly suggest a sensorimotor interpretation. Activation is preponderantly in the right hemisphere, including areas of visual cortex and prefrontal cortex. Furthermore, spatial working memory and object working memory are subserved by different brain regions, with spatial working memory activating more dorsal areas than object working memory and also activating prefrontal areas involved in spatial attention (see E. E. Smith, 2000, for review).

These findings are balanced by evidence of lefthemisphere involvement in verbal working memory, including known speech processing areas. One source of such evidence is the dissociations of abilities found in neurological patients. Various studies have reported patients who appear to have defective storage but intact rehearsal, often accompanying lesions in the left temporoparietal area, whereas other patients appear to have intact storage but defective rehearsal, often accompanying lesions in the left frontal area near Broca's area and supplementary motor cortex (Allport, 1984; Baeta \& Ferro, 1988; Basso, Spinnler, Vallar, \& Zanobio, 1982; Belleville, Peretz, \& Arguin, 1992; Howard \& Franklin, 1993; Maeshima, Uematsu, Ozaki, \& Fujita, 1997; Nickels, Howard, \& Best, 1997; Shallice \& Butterworth, 1977; Shallice \& Vallar, 1990; Vallar \& Baddeley, 1984; Vallar \& Cappa, 1987; Vallar, Di Betta, \& Silveri, 1997; Warrington \& Shallice, 1969; Waters, Rochon, \& Caplan, 1992). The dissociations observed between these components, as well as the involvement of sites known to play roles in phonological processing and speech planning, respectively, support a sensorimotor account of storage and rehearsal.

However, the pattern of data in patients with working memory deficits is not always fully consistent with damage to one or the other of the two hypothesized components. Waters et al. (1992), for instance, report six apraxic patients whose deficits are broadly consistent with disrupted rehearsal, but who show important individual differences in the precise pattern of disrupted and spared effects. Similar deviations from the predicted patterns can be found throughout the literature on this topic. One source of these inconsistencies certainly is the variability that exists in the size and location of lesions, making it 
difficult to find pure cases of disruption of a single cognitive mechanism. However, a clue to another possible cause is provided by Cubelli and Nichelli (1992), who propose the existence of two distinct articulatory mechanisms that serve independent functions within working memory.

Cubelli and Nichelli (1992) report 2 anarthric patients (patients incapable of speech) whose speech deficits are caused by damage to two distinct brain regions: Patient C.M. suffered bilateral damage to the pons, resulting in "locked-in" syndrome, whereas Patient F.C. suffered damage to the left precentral gyrus near Broca's area, resulting in cortical anarthria. These two forms of anarthria were accompanied by disruption of two distinct functions of articulation in working memory.

Patient C.M. showed no word length effect but did show a phonological similarity effect even with visual presentation. This pattern suggests loss of the ability to perform rehearsal but retained ability to recode print into the phonological code used by the store. (We should note that disruption in rehearsal due to anarthria is not incompatible with the finding of preserved rehearsal in congenital anarthrics, since the disruption of rehearsal will depend on how peripheral the deficit is. Several authors have argued that rehearsal will be disrupted only by damage to speech planning mechanisms and not by damage to peripheral speech production mechanisms [e.g., Baddeley $\&$ Wilson, 1985]. When disruption does occur, though, the particular pattern of disruption can be analyzed for its bearing on the structure of working memory.)

In contrast, Patient F.C. showed both a length effect and a similarity effect with auditory presentation but showed neither effect when presentation was visual. This suggests loss of the ability to recode from print but spared ability to rehearse. The authors also review previous reports of anarthric patients with either pontine or frontal cortical damage, which show that this pattern occurs consistently (e.g., Baeta \& Ferro, 1988; Vallar \& Cappa, 1987). Martin, Blossom-Stach, Yaffee, and Wetzel (1995) report a patient with damage to the left frontotemporal region and impairment of motor programming. This patient, like F.C., displays exactly the pattern expected when recoding but not rehearsal is disrupted, although the authors do not discuss the results in this light.

On the basis of these data, Cubelli and Nichelli (1992) propose that articulatory recoding and articulatory rehearsal are two distinct functions of articulation within working memory and are subserved by distinct anatomical regions (see Monsell, 1987, for a similar proposal). Note that these two functions have both been discussed in the behavioral literature but have not been dissociated from one another. Rehearsal, which is used for maintenance and is part of the backbone of working memory, and recoding, which is necessary only when nonphonological materials must be translated into phonological form prior to rehearsal, are both disrupted by articulatory suppression and hence have been assumed to reflect one and the same articulatory process. Cubelli and Nichelli's data suggest that in fact distinct parts of the brain's articulatory machinery subserve these two functions.

While the literature on damage to working memory is generally supportive of the sensorimotor model, though somewhat messy in its details, a clearer view of the neural substrates of verbal working memory can be found in the literature on brain imaging. Using PET imaging, Awh et al. (1996) found evidence for anatomically separate storage and rehearsal components (see also Fiez et al., 1996; Jonides et al., 1998). In Awh et al.'s first experiment, subjects indicated whether a probe letter was one of four letters presented $3 \mathrm{sec}$ earlier. A letter matching task, in which the four letters were still present when the probe appeared, served as a baseline for subtraction. Activation was found in areas involved in language production (Broca's area, left premotor cortex, left supplementary motor cortex, and right cerebellum), as well as in left posterior parietal cortex, which has been implicated in phonological storage.

In a second experiment, Awh et al. (1996) confirmed these findings with a different task and additionally disentangled the functions of storage and rehearsal. Subjects performed a "2-back" task in which each of a continuous sequence of letters must be compared with the letter that occurred two items previously. Although this task is probably a relatively impure verbal maintenance task, since it involves repeatedly updating the positions and identities of the items to be remembered, it has the advantage of engaging subjects continuously in maintenance. When a condition in which subjects simply monitored the same stimulus sequence for a particular letter was used as a baseline for subtraction, activation was found in all the areas identified in Experiment 1 (as well as in additional areas in the right hemisphere whose roles in the task are unclear). When, instead, the baseline condition consisted of subjects subvocally repeating each letter until the next appeared, the activation in Broca's area and premotor cortex dropped out. This implicates these areas specifically in the rehearsal portion of the memory maintenance task, leaving the remaining areas-notably, left posterior parietal cortex - as candidates for the storage component (see also Jonides et al., 1998). (Note, though, that the "rehearsal" control would involve both of the two hypothesized functions of articulation, so that we cannot distinguish their neural substrates on the basis of these data.)

Further support for these conclusions comes from a PET study by Paulesu, Frith, and Fackowiak (1993). In a task similar to Awh et al.'s (1996) first experiment, subjects were shown a sequence of six letters and then asked whether a probe letter was one of the sequence. In this case, however, the comparison condition was the same memory task using unfamiliar Korean letters that could not be recoded phonologically. Bilateral activation was found in several areas, but the level of activation was asymmetric between the two hemispheres, favoring Broca's area, the left supramarginal gyrus, and the right cerebellum. (Differential activation was also found in left Brod- 
man's areas 22/42 and 18, which have been implicated, respectively, in passive perception of phonemes and in visual processing of familiar letters. These functions would not have been subtracted out by the Korean letter control task.) A second experiment used a rhyming task, which is thought to involve the articulatory component of working memory but does not involve memory storage. The control task in this case was a visual similarity judgment for Korean letters. In a statistical comparison with Experiment 1, the only area to show less activation in Experiment 2 was the left supramarginal gyrus, suggesting that this area may be involved in the storage component (see Becker, MacAndrew, \& Fiez, 1999, for critique concerning the precise localization of the storage component).

Thus, the data from brain damage and brain imaging suggest that articulation and phonological storage are indeed two separate functions of verbal working memory, subserved by distinct anatomical areas. Furthermore, the brain areas involved include sites known to play roles in speech output planning, such as Broca's area and the cerebellum, as well as sites involved in phonological processing at or near Wernicke's area. In addition, the data suggest the existence of two separate articulatory functions involving two distinct brain areas, which are both blocked by articulatory suppression and hence have not been distinguished from one another in the behavioral data.

\section{Conclusions}

A wide range of phenomena, then, point toward the use of sensorimotor coding in working memory. These include not only the traditional effects, whose behavior taken as an overall pattern is difficult to explain in other terms, but also striking additional findings, such as the errors produced by children with speech impediments and the modality-specific quality of sign language rehearsal. Furthermore, findings that have been considered to be in conflict with the sensorimotor account, such as the word length effect in congenital anarthrics and the apparent interaction between word length and irrelevant speech, can in fact be accommodated by this account without great difficulty. Although models that do not appeal to sensorimotor coding have been successful in explaining portions of the data, the entire body of data taken as a whole can be captured only by some variant of a sensorimotor model.

In addition, it is worth noting that the use of sensorimotor coding in working memory accords with introspective experience. Psychology teachers routinely ask their students how they would remember a phone number long enough to cross the room and dial a phone, and students routinely reply, "I would say it to myself." A variety of other everyday tasks yield the same intuition: keeping track of the next few ingredients for a recipe; memorizing a shopping list, at least until it starts to "stick"; planning what to say in an upcoming situation; temporarily keeping track of a few digits in the middle of long division; "reviewing" a complex or obscure sentence to work out what it means. Although introspective evidence should certainly be viewed cautiously, such a vivid, specific, and nearly universal subjective impression calls for explanation. It is one more point in favor of the sensorimotor approach that it accounts for the phenomenology of rehearsal.

This conclusion also has the advantage of positing mechanisms similar to those that operate in nonlinguistic visuospatial working memory, thus adding coherence and explanatory simplicity to the overall concept of working memory. In both the linguistic case and the nonlinguistic case, peripheral sensorimotor mechanisms are at work, allowing the cognitive system to temporarily "off-load" information until it is needed. It is the special structural characteristics of language - perhaps including how highly overlearned it is, or how tight and automatic the mapping is between input and output representations-that are responsible for the rehearsal loop structure of linguistic working memory observed for both speech and sign. The underlying basis of this kind of rehearsal loop, however, appears to be the same as for nonlinguistic memory-the recruitment of sensory and motor resources in order to "embody" information for temporary storage.

\section{REFERENCES}

Allport, D. A. (1984). Auditory-verbal short-term memory and conduction aphasia. In H. Bouma \& D. G. Bouwhuis (Eds.), Attention and performance X: Control of language processes (pp. 313-325). Hillsdale, NJ: Erlbaum.

Avons, S. E., Wright, K. L., \& Pammer, K. (1994). The word-length effect in probed and serial recall. Quarterly Journal of Experimental Psychology, 47A, 207-231.

Awh, E., Jonides, J., Smith, E. E., Buxton, R. B., Frank, L. R., Love, T., Wong, E. C., \& GMeIndL, L. (1999). Rehearsal in spatial working memory: Evidence from neuroimaging. Psychological Science, 10, 433-437.

Awh, E., Jonides, J., Smith, E. E., Schumacher, E. H., Koeppe, R. A., \& KATZ, S. (1996). Dissociation of storage and rehearsal in verbal working memory: Evidence from positron emission tomography. Psychological Science, 7, 25-31.

BADDELEY, A. D. (1986). Working memory. Oxford: Oxford University Press.

BAdDeley, A. D. (1990). The development of the concept of working memory: Implications and contributions of neuropsychology. In G. Vallar \& T. Shallice (Eds.), Neuropsychological impairments of short-term memory (pp. 54-73). New York: Cambridge University Press.

BAdDeley, A. D. (1995). Working memory. In M. S. Gazzaniga (Ed.), The cognitive neurosciences (pp. 755-764). Cambridge, MA: MIT Press.

Baddeley, A. D. (2000). The phonological loop and the irrelevant speech effect: Some comments on Neath (2000). Psychonomic Bulletin \& Review, 7, 544-549.

BADDElEY, A. D., \& ANDRADE, J. (1994). Reversing the word-length effect: A comment on Caplan, Rochon, and Waters. Quarterly Journal of Experimental Psychology, 47A, 1047-1054.

Baddeley, A. D., Gathercole, S., \& Papagno, C. (1998). The phonological loop as a language learning device. Psychological Review, 105, 158-173.

Baddeley, A. D., \& Hitch, G. (1974). Working memory. In G. H. Bower (Ed.), The psychology of learning and motivation (Vol. 8, pp. 647-667). Hillsdale, NJ: Erlbaum.

BAdDeley, A. D., \& Hitch, G. (1994). Developments in the concept of working memory. Neuropsychology, 8, 485-493.

Baddeley, A. D., Lewis, V. J., \& VAllar, G. (1984). Exploring the articulatory loop. Quarterly Journal of Experimental Psychology, 36A, 233-252.

Baddeley, A. D., Thomson, N., \& Buchanan, M. (1975). Word length 
and the structure of short-term memory. Journal of Verbal Learning \& Verbal Behavior, 14, 575-589.

BADDEley, A. D., \& Wilson, B. (1985). Phonological coding and shortterm memory in patients without speech. Journal of Memory \& Language, 24, 490-502.

Baeta, E., \& Ferro, J. M. (1988, July). Phonological study in an anarthric patient. Paper presented at the 11th European Conference of the INS, Lathi, Finland.

Basso, A., Spinnler, H., Vallar, G., \& Zanobio, M. E. (1982). Left hemisphere damage and selective impairment of auditory verbal short-term memory: A case study. Neuropsychologia, 20, 263-274.

Becker, J. T., MacAndrew, D. K., \& Fiez, J. A. (1999). A comment on the functional localization of the phonological storage subsystem of working memory. Brain \& Cognition, 41, 27-38.

Belleville, S., Peretz, I., \& Arguin, M. (1992). Contribution of articulatory rehearsal to short-term memory: Evidence from a case of selective disruption. Brain \& Language, 43, 713-746.

Bishop, D. V. M., \& Robson, J. (1989). Unimpaired short-term memory and rhyme judgment in congenitally speechless individuals: Implications for the notion of "articulatory coding." Quarterly Journal of Experimental Psychology, 41A, 123-141.

Boyle, R., \& Coltheart, V. (1996). Effects of irrelevant sounds on phonological coding in reading comprehension and short-term memory. Quarterly Journal of Experimental Psychology, 49A, 398-416.

BRENTARI, D. (1990). Theoretical foundations of American Sign Language phonology. Unpublished doctoral dissertation, University of Chicago.

Brown, G. D. A., \& Hulme, C. (1995). Modeling item length effects in memory span: No rehearsal needed? Journal of Memory \& Language, 34, 594-621.

Burgess, N., \& Hitch, G. J. (1992). Toward a network model of the articulatory loop. Journal of Memory \& Language, 31, 429-460.

Burgess, N., \& Hitch, G. J. (1999). Memory for serial order: A network model of the phonological loop and its timing. Psychological Review, 106, 551-581.

CANTOR, J., \& ENGLE, R. W. (1993). Working-memory capacity as long-term memory activation: An individual-differences approach. Journal of Experimental Psychology: Learning, Memory, \& Cognition, 19, 1101-1114.

Caplan, D., Rochon, E., \& Waters, G. S. (1992). Articulatory and phonological determinants of word length effects in span tasks. Quarterly Journal of Experimental Psychology, 45A, 177-192.

Caplan, D., \& Waters, G. S. (1994). Articulatory length and phonological similarity in span tasks: A reply to Baddeley and Andrade. Quarterly Journal of Experimental Psychology, 47A, 1055-1062.

Carpenter, P. A., Miyake, A., \& Just, M. A. (1994). Working memory constraints in comprehension: Evidence from individual differences, aphasia, and aging. In M. A. Gernsbacher (Ed.), Handbook of psycholinguistics (pp. 1075-1122). San Diego: Academic Press.

Clark, A. (1997). Being there: Putting brain, body, and world together again. Cambridge, MA: MIT Press.

Coltheart, V., \& Langdon, R. (1998). Recall of short word lists presented visually at fast rates: Effects of phonological similarity and word length. Memory \& Cognition, 26, 330-342.

Corina, D., \& Sandler, W. (1993). On the nature of phonological structure in sign language. Phonology, 10, 165-207.

Coulter, G. R. (ED.) (1993). Phonetics and phonology: Current issues in ASL phonology. San Diego: Academic Press.

Cowan, N., Day, L., Saults, J. S., Keller, T. A., Johnson, T., \& FloRES, L. (1992). The role of verbal output time in the effects of word length on immediate memory. Journal of Memory \& Language, 31, 1-17.

Cowan, N., Keller, T., Hulme, C., Roodenrys, S., McDougall, S., \& RACK, J. (1994). Verbal memory span in children: Speech timing clues to the mechanisms underlying age and word length effects. Journal of Memory \& Language, 33, 234-250.

Cowan, N., Nugent, L. D., Elliott, E. M., \& Geer, T. (2000). Is there a temporal basis of the word length effect? A response to Service (1998). Quarterly Journal of Experimental Psychology, 53A, 661-665.

Cowan, N., Wood, N. L., Nugent, L. D., \& Treisman, M. (1997).
There are two word-length effects in verbal short-term memory: Opposed effects of duration and complexity. Psychological Science, $\mathbf{8}$, 290-295.

Cowan, N., Wood, N. L., Wood, P. K., Keller, T. A., Nugent, L. D., \& Keller, C. V. (1998). Two separate verbal processing rates contributing to short-term memory span. Journal of Experimental Psychology: General, 127, 141-160.

Cubelli, R, \& Nichelli, P. (1992). Inner speech in anarthria: Neuropsychological evidence of differential effects of cerebral lesions on subvocal articulation. Journal of Clinical \& Experimental Neuropsychology, 14, 499-517.

Ellis, N. C., \& Hennelly, R. A. (1980). A bilingual word-length effect: Implications for intelligence testing and the relative ease of mental calculation in Welsh and English. British Journal of Psychology, 71, 43-52.

Ellis, N. C., \& Sinclair, S. G. (1996). Working memory in the acquisition of vocabulary and syntax: Putting language in good order. Quarterly Journal of Experimental Psychology, 49A, 234-250.

Fiez, J. A., Raife, E. A., Balota, D. A., Schwarz, J. P., Raichle, M. E., \& Petersen, S. E. (1996). A positron emission tomography study of the short-term maintenance of verbal information. Journal of Neuroscience, 16, 808-822.

Gathercole, S. E., Adams, A.-M., \& Hitch, G. J. (1994). Do young children rehearse? An individual-differences analysis. Memory \& Cognition, 22, 201-207.

Gathercole, S. E., \& Baddeley, A. D. (1993). Working memory and language. Hillsdale, NJ: Erlbaum.

Gathercole, S. E., \& Martin, A. J. (1996). Interactive processes in phonological memory. In S. E. Gathercole (Ed.), Models of shortterm memory (pp. 73-100). Hove, U.K.: Psychology Press.

GlenberG, A. M. (1997). What memory is for. Behavioral \& Brain Sciences, 20, 1-55.

GlenberG, A. M., \& Swanson, N. G. (1986). A temporal distinctiveness theory of recency and modality effects. Journal of Experimental Psychology: Learning, Memory, \& Cognition, 12, 3-15.

Gupta, P., \& MAcWhinney, B. (1995). Is the articulatory loop articulatory or auditory? Reexamining the effects of concurrent articulation on immediate serial recall. Journal of Memory \& Language, 34, 63-88

Hanley, J. R., \& Broadbent, C. (1987). The effect of unattended speech on serial recall following auditory presentation. British Journal of Psychology, 78, 287-297.

Henson, R. A. (1998). Short-term memory for serial order: The startend model. Cognitive Psychology, 36, 73-137.

Hitch, G. J., \& BADDELEY, A. D. (1976). Verbal reasoning and working memory. Quarterly Journal of Experimental Psychology, 28, 603-621.

Howard, D., \& Butterworth, B. (1989). Short-term memory and sentence comprehension: A reply to Vallar and Baddeley, 1987. Cognitive Psychology, 6, 455-463.

HowARD, D., \& Franklin, S. (1993). Dissociations between component mechanisms in short-term memory: Evidence from brain-damaged patients. In D. E. Meyer \& S. Kornblum (Eds.), Attention and performance XIV: Synergies in experimental psychology, artificial intelligence, and cognitive neuroscience (pp. 425-449). Cambridge, MA: MIT Press.

Johnson, M. (1987). The body in the mind: The bodily basis of imagination, reason, and meaning. Chicago: University of Chicago Press.

Jones, D. M., BeAman, P., \& Macken, W. J. (1996). The object-oriented episodic record model. In S. E. Gathercole (Ed.), Models of shortterm memory (pp. 209-237). Hove, U.K.: Psychology Press.

Jones, D. M., \& MACKen, W. J. (1993). Irrelevant tones produce an irrelevant speech effect: Implications for phonologically coding in working memory. Journal of Experimental Psychology: Learning, Memory, \& Cognition, 19, 369-381.

Jones, D. M., Macken, W. J., \& Murray, A. C. (1993). Disruption of visual short-term memory by changing-state auditory stimuli: The role of segmentation. Memory \& Cognition, 21, 318-328.

Jones, D. M., \& Tremblay, S. (2000). Interference in memory by process or content? A reply to Neath (2000). Psychonomic Bulletin \& Review, 7, 550-558. 
Jonides, J., Schumacher, E. H., Smith, E. E., Koeppe, R. A., Awh, E., Reuter-Lorenz, P. A., Marshuetz, C., \& Willis, C. R. (1998). The role of parietal cortex in verbal working memory. Journal of Neuroscience, 18, 5026-5034.

Jonides, J., \& SMith, E. E. (1997). The architecture of working memory. In M. D. Rugg (Ed.), Cognitive neuroscience (pp. 243-276). Cambridge, MA: MIT Press.

Keller, T. A., Cowan, N., \& Saults, J. S. (1995). Can auditory memory for tone pitch be rehearsed? Journal of Experimental Psychology: Learning, Memory, \& Cognition, 21, 635-645.

Klima, E. S., \& Bellugi, U. (1979). The signs of language. Cambridge, MA: Harvard University Press.

KraKow, R. A., \& HANSON, V. L. (1985). Deaf signers and serial recall in the visual modality: Memory for signs, fingerspelling, and print. Memory \& Cognition, 13, 265-272.

Kutas, M., \& Federmeier, K. D. (1998). Minding the body. Psychophysiology, 35, 135-150.

Lakoff, G., \& Johnson, M. (1999). Philosophy in the flesh: The embodied mind and its challenge to Western thought. New York: Basic Books.

LeCompte, D. C. (1996). Irrelevant speech, serial rehearsal, and temporal distinctiveness: A new approach to the irrelevant speech effect. Journal of Experimental Psychology: Learning, Memory, \& Cognition, 22, 1154-1165.

LeCompte, D. C., Neely, C. B., \& Wilson, J. R. (1997). Irrelevant speech and irrelevant tones: The relative importance of speech to the irrelevant speech effect. Journal of Experimental Psychology: Learning, Memory, \& Cognition, 23, 472-483.

LeCompte, D. C., Speed, A. E., \& Marks, A. R. (2000). Faster than you can say "rehearsal": An irrelevant speech effect with rapidlypresented lists. Unpublished manuscript.

LI, S., \& LEWANDOWSKI, S. (1993). Intralist distractors and recall direction: Constraints on models of memory for serial order. Journal of Experimental Psychology: Learning, Memory, \& Cognition, 19, 895 908.

LI, S., \& LEWANDOWSKI, S. (1995). Forward and backward recall: Different retrieval processes. Journal of Experimental Psychology: Learning, Memory, \& Cognition, 21, 837-847.

Liberman, A. M. (1996). Speech: A special code. Cambridge, MA: MIT Press.

Locke, J. L., \& Kutz, K. J. (1975). Memory for speech and speech for memory. Journal of Speech \& Hearing Research, 18, 176-191.

LogIE, R. H. (1995). Visuo-spatial working memory. Hillsdale, NJ: Erlbaum.

Longoni, A. M., Richardson, J. T. E., \& Aiello, A. (1993). Articulatory rehearsal and phonological storage in working memory. Memory \& Cognition, 21, 11-22.

MACKEN, W. J., \& JonES, D. M. (1995). Functional characteristics of the inner voice and the inner ear: Single or double agency? Journal of Experimental Psychology: Learning, Memory, \& Cognition, 21, 436448 .

Maeshima, S., Uematsu, Y., Ozaki, F., \& Fujita, K. (1997). Impairment of short-term memory in left hemispheric traumatic brain injuries. Brain Injury, 11, 279-286.

Marks, A. R., \& Crowder, R. G. (1997). Temporal distinctiveness and modality. Journal of Experimental Psychology: Learning, Memory, \& Cognition, 23, 164-180.

Marschark, M. (1996, November). Influences of signed and spoken language on memory span. Paper presented at the annual meeting of the Psychonomic Society, Chicago.

Martin, R. C. (1995). Working memory doesn't work: A critique of Miyake et al.'s capacity theory of aphasic comprehension deficits. Cognitive Neuropsychology, 12, 623-636.

Martin, R. C., Blossom-Stach, C., Yaffee, L. S., \& Wetzel, W. F. (1995). Consequences of a motor programming deficit for rehearsal and written sentence comprehension. Quarterly Journal of Experimental Psychology, 48A, 536-572.

Martin, R. C., \& LeSCH, M. F. (1996). Associations and dissociations between language impairment and list recall: Implications for models of STM. In S. Gathercole (Ed.), Models of short-term memory (pp. 149-178). Hove, U.K.: Psychology Press.
Martin, R. C., \& Romani, C. (1994). Verbal working memory and sentence comprehension: A multiple-components view. Neuropsychology, 8, 506-523.

Martin, R. C., Shelton, J. R., \& Yaffee, L. S. (1994). Language processing and working memory: Neuropsychological evidence for separate phonological and semantic capacities. Journal of Memory \& Language, 33, 83-111.

Mayberry, R, \& Eichen, E. (1991). The long-lasting advantage of learning sign language in childhood: Another look at the critical period for language acquisition. Journal of Memory \& Language, 30, 486-512.

Meltzoff, A. N., \& Moore, M. K. (1995). Infants' understanding of people and things: From body imitation to folk psychology. In J. L. Bermúdez, A. Marcel, \& N. Eilan (Eds.), The body and the self (pp. 43-69). Cambridge, MA: MIT Press.

Monsell, S. (1987). On the relation between lexical input and output pathways for speech. In A. Allport, D. G. MacKay, \& W. Prinz (Eds.), Language perception and production: Relationships between listening, speaking, reading and writing (pp. 273-311). London: Academic Press.

NAIRne, J. S. (1990). A feature model of immediate memory. Memory \& Cognition, 18, 251-269.

Nairne, J. S., \& Kelley, M. R. (1999). Reversing the phonological similarity effect. Memory \& Cognition, 27, 45-53.

NeATH, I. (2000). Modeling the effects of irrelevant speech on memory. Psychonomic Bulletin \& Review, 7, 403-423.

Neath, I., Surprenant, A. M., \& LeCompte, D. C. (1998). Irrelevant speech eliminates the word length effect. Memory \& Cognition, 26, 343-354.

Neely, C. B., \& LeCompte, D. C. (1999). The importance of semantic similarity to the irrelevant speech effect. Memory \& Cognition, 27, 37-44.

Nickels, L., Howard, D., \& Best, W. (1997). Fractionating the articulatory loop: Dissociations and associations in phonological recoding in aphasia. Brain \& Language, 56, 161-182.

PAGe, M. P. A., \& Norris, D. (1998). The primacy model: A new model of immediate serial recall. Psychological Review, 105, 761-781.

Paulesu, E., Bottini, G., \& Frackowiak, R. S. (1997). Cognitiveneurology and the contribution of neuroimaging. In M. R. Trimble \& J. L. Cummings (Eds.), Contemporary behavioral neurology (Blue Books of Practical Neurology, Vol. 16, pp. 49-89). Boston, MA: ButterworthHeinemann.

Paulesu, E., Frith, C. D., \& Frackowiak, R. S. (1993). The neural correlates of the verbal component of working memory. Nature, $\mathbf{3 6 2}$, 342-345.

Pechmann, T., \& Mohr, G. (1992). Interference in memory for tonal pitch: Implications for a working-memory model. Memory \& Cognition, 20, 314-320.

Poizner, H., Bellugi, U., \& Tweney, R. (1981). Processing of formational, semantic, and iconic information in American Sign Language. Journal of Experimental Psychology: Human Perception \& Performance, 7, 1146-1159.

QuinN, J. G., \& McConnell, J. (1996). Irrelevant pictures in visual working memory. Quarterly Journal of Experimental Psychology, 49A, 200-215.

Richardson, J. T. E., Engle, R. W., Hasher, L., Logie, R. H., StoltzFUS, E. R., \& ZACKS, R. T. (Eds.) (1996). Working memory and human cognition. New York: Oxford University Press.

SAlAmÉ, P., \& BADDELEY, A. (1982). Disruption of short-term memory by unattended speech: Implications for the structure of working memory. Journal of Verbal Learning \& Verbal Behavior, 21, 150-164.

SANDLER, W. (1989). Phonological representation of the sign: Linearity and nonlinearity in American Sign Language. Dordrecht: Foris.

Scatena, P. (1990). Phantom representations of congenitally absent limbs. Perceptual \& Motor Skills, 70, 1227-1232.

SCHWEICKERT, R, \& BORUFF, B. (1986). Short-term memory capacity: Magic number or magic spell? Journal of Experimental Psychology: Learning, Memory, \& Cognition, 12, 419-425.

Schweickert, R., Guentert, L., \& Hersberger, L. (1990). Phonological similarity, pronunciation rate, and memory span. Psychological Science, 1, 74-77. 
Serra, M., \& Jonas, D. L. (1996, November). Evaluating the dual process account of forward and backward serial recall. Paper presented at the annual meeting of the Psychonomic Society, Chicago.

Service, E. (1998). The effect of word length on immediate serial recall depends on phonological complexity, not articulatory duration. Quarterly Journal of Experimental Psychology, 51A, 283-304.

Shallice, T., \& Butterworth, B. (1977). Short-term memory impairment and spontaneous speech. Neuropsychologia, 15, 729-735.

Shallice, T., \& Vallar, G. (1990). The impairment of auditory-verbal short-term storage. In G. Vallar \& T. Shallice (Eds.), Neuropsychological impairments of short-term memory (pp. 11-53). New York: Cambridge University Press.

Shand, M. A., \& KLIMA, E. S. (1981). Nonauditory suffix effects in congenitally deaf signers of American sign language. Journal of Experimental Psychology: Human Learning \& Memory, 7, 464-474.

Smith, E. E. (2000). Neural bases of human working memory. Current Directions in Psychological Science, 9, 45-49.

Smith, E. E., \& JonidES, J. (1997). Working memory: A view from neuroimaging. Cognitive Psychology, 33, 5-42.

Smith, J. D., Reisberg, D., \& Wilson, M. (1992). Subvocalization and auditory imagery: Interactions between the inner ear and inner voice. In D. Reisberg (Ed.), Auditory imagery (pp. 95-119). Hillsdale, NJ: Erlbaum.

Smith, J. D., Wilson, M., \& Reisberg, D. (1995). The role of inner speech in auditory imagery. Neuropsychologia, 33, 1433-1454.

Stigler, J. W., Lee, S. Y., \& Stevenson, H. W. (1986). Digit memory in Chinese and English: Evidence for a temporally limited store. Cognition, 23, 1-20.

Surprenant, A. M., Neath, I., \& LeCompte, D. C. (1999). Irrelevant speech, phonological similarity, and presentation modality. Memory, 7, 405-420.

Tremblay, S., Macken, W. J., \& Jones, D. M. (1999, November). Elimination of the word length effect by irrelevant sound revisited. Paper presented at the annual meeting of the Psychonomic Society, Los Angeles.

VAllar, G., \& BAdDEley, A. (1984). Fractionation of working memory: Neuropsychological evidence for a short-term store. Journal of Verbal Learning \& Verbal Behavior, 23, 151-161.

VAllar, G., \& CAPPA, S. F. (1987). Articulation and verbal short-term memory: Evidence from anarthria. Cognitive Neuropsychology, 4, 55-77.

Vallar, G., Di Betta, A. M., \& Silveri, M. C. (1997). The phonological short-term store-rehearsal system: Patterns of impairment and neural correlates. Neuropsychologia, 35, 795-812.

Vallar, G., \& Papagno, C. (1995). Neuropsychological impairments of short-term memory. In A. D. Baddeley, B. A. Wilson, \& F. N. Watts (Eds.), Handbook of memory disorders (pp. 135-165). New York: Wiley.

VAllar, G., \& Shallice, T. (1990). Neuropsychological impairments of short-term memory. New York: Cambridge University Press.

Warrington, E. K., \& Shallice, T. (1969). The selective impairment of auditory verbal short-term memory. Brain, 92, 885-896.

Waters, G. S., Rochon, E., \& Caplan, D. (1992). The role of highlevel speech planning in rehearsal: Evidence from patients with apraxia of speech. Journal of Memory \& Language, 31, 54-73.

Wilson, M., Bettger, J. G., Niculae, I., \& Klima, E. S. (1997). Modality of language shapes working memory: Evidence from digit span and spatial span in ASL signers. Journal of Deaf Studies \& Deaf Education, 2, 150-160.

Wilson, M., \& Emmorey, K. (1997a). A visuospatial "phonological loop" in working memory: Evidence from American Sign Language. Memory \& Cognition, 25, 313-320.

Wilson, M., \& EMmorey, K. (1997b). Working memory for sign language: A window into the architecture of working memory. Journal of Deaf Studies \& Deaf Education, 2, 123-132.

Wilson, M., \& Emmorey, K. (1998a, November). Spatial coding in working memory for signs. Paper presented to the Sixth International Conference on Theoretical Issues in Sign Language Research, Gallaudet University, Washington, DC.

Wilson, M., \& EMmorey, K. (1998b). A “word length effect" for sign language: Further evidence for the role of language in structuring working memory. Memory \& Cognition, 26, 584-590.

Wilson, M., Iverson, A., \& EMmorey, K. (2001). Modality matters: Spatial coding in working memory for sign language. Manuscript under review.

(Manuscript received July 14, 1999; revision accepted for publication June 12, 2000.) 